\title{
Use of a web portal by adult patients with pre-diabetes and type 2 diabetes mellitus seen in a family medicine outpatient clinic
}

\author{
Steven S. Coughlin ${ }^{1,2}$, Vahé Heboyan ${ }^{1}$, Lufei Young ${ }^{3}$, Gianluca De Leo ${ }^{1}$, Thad Wilkins ${ }^{4}$ \\ ${ }^{1}$ Department of Clinical and Digital Health Sciences, College of Allied Health Sciences, Augusta University, Augusta, GA, USA; ${ }^{2}$ Research Service, \\ Charlie Norwood Veterans Affairs Medical Center, Augusta, GA, USA; ${ }^{3}$ College of Nursing, ${ }^{4}$ Department of Family Medicine, Medical College of \\ Georgia, Augusta University, Augusta, GA, USA \\ Contributions: (I) Conception and design: SS Coughlin; (II) Administrative support: SS Coughlin; (III) Provision of study materials: SS Coughlin; (IV) \\ Collection and assembly of data: SS Coughlin; (V) Data analysis and interpretation: V Heboyan; (VI) Manuscript writing: All authors; (VII) Final \\ approval of manuscript: All authors. \\ Correspondence to: Dr. Steven S. Coughlin. Associate Professor, Clinical and Digital Health Sciences, College of Allied Health Sciences, Augusta \\ University, $112015^{\text {th }}$ Street, EC-4324, Augusta, GA 30912, USA. Email: scoughlin@augusta.edu.
}

\begin{abstract}
Background: There has been increasing interest in the use of web portals by patients with type 2 diabetes mellitus (T2DM). Studies of web portal use by patients with pre-diabetes have not been reported. To plan studies of web portal use by adult clinic patients seen for pre-diabetes and T2DM at an academic medical center, we examined characteristics of those who had or had not registered for a web portal.

Methods: Electronic records were reviewed to identify web portal registration by patients treated for prediabetes or T2DM by age, sex, race and ethnicity.

Results: A total of 866 patients with pre-diabetes and 2,376 patients with T2DM were seen in a family medicine outpatient clinic. About $41.5 \%$ of patients with pre-diabetes and $34.7 \%$ of those with T2DM had registered for the web portal. In logistic regression analysis, web portal registration among patients with T2DM was significantly associated with age 41-45 years, and with Hispanic ethnicity. Similar results were obtained for pre-diabetes except that the positive association with age 41-45 years and inverse association with Hispanic ethnicity were not statistically significant. Among patients with pre-diabetes or T2DM, Black men and Black women were less likely to have registered than their white counterparts. Patients who were aged 18-25 and >65 years were less likely to have registered for the web portal than those 26-65 years.

Conclusions: Additional research is needed to identify portal design features that improve health outcomes for patients with pre-diabetes and T2DM and interventions that will increase use of patient portals by pre-diabetic and diabetic patients, especially among Black patients and older patients.
\end{abstract}

Keywords: Blacks; diabetes; electronic health record; glycemic control; health information technology; impaired glucose tolerance; patient web portals

Received: 19 March 2018; Accepted: 13 April 2018; Published: 09 May 2018.

doi: 10.21037/jhmhp.2018.04.04

View this article at: http://dx.doi.org/10.21037/jhmhp.2018.04.04

\section{Introduction}

Over 30 million Americans had diabetes in 2015 (1). An additional $35 \%$ of U.S. adults have pre-diabetes (impaired glucose tolerance or impaired fasting glucose) which is associated with a four-fold increased risk of progressing to diabetes compared to those who are normo-glycemic (2).
Persons with diabetes have an increased risk of macrovascular and microvascular complications (3). Good glycemic control is associated with reduced morbidity and mortality, but almost half of patients with type 2 diabetes mellitus (T2DM) do not meet recommended targets for glycemic control (4-6).

The management of $\mathrm{T} 2 \mathrm{DM}$ requires effective 
communication between providers and patients and patient self-management (7). Studies have demonstrated that increasing patients' knowledge about their risk factors can lead to improved clinical outcomes $(8,9)$. For both prediabetic and diabetic patients, fostering a collaborative relationship between patients and their health care providers is likely to improve clinical outcomes $(10,11)$.

Efforts at health care reform and continued advances in information technologies have increased interest in patient web portals. Stakeholders view patient portals, and other technological advances such as personal health records and electronic medical records (EMRs), as an opportunity to leverage information technology to support patient self-management and improve communication between patients and providers $(12,13)$. The introduction of patient web portals constitutes an important development in prediabetes and diabetes care.

The goal of the current feasibility study was to determine the number of adult patients seen for pre-diabetes or T2DM in a family medicine clinic at a large academic medical center in the southeastern U.S. and to examine whether they had registered for the patient web portal according to age, sex, race and Hispanic ethnicity.

\section{Methods}

\section{Ethics approval}

The current study was conducted as "preparatory to research" in support of grant applications for larger and more detailed proposed studies. Prior to a review of computerized patient records, a determination was made that the study was exempt from IRB review. Informed consent was not required for a review of existing patient records that did not include any personally identifying information.

\section{Methods}

EMRs were reviewed including web portal registration by adult patients treated for pre-diabetes (impaired glucose tolerance and impaired fasting glucose) or T2DM. The patients were seen in a family medicine clinic at a large academic health center. De-identified number of patients seen for pre-diabetes and T2DM were examined by age categories, sex, race and ethnicity (Table 1). Odds ratios, 95\% confidence intervals (CI), and $\mathrm{P}$ values were obtained using STATA Data Analysis and
Statistical Software. An approximate chi-square test was used to test for interaction on an additive scale (14). Multivariate analysis was carried out using logistic regression methods to determine adjusted odds ratios and assess statistical significance (15). In this regression method, the logarithmic odds of disease is modeled as a linear function of explanatory variables and maximum likelihood estimates of their beta coefficients.

\section{Results}

A total of 866 patients with pre-diabetes and 2,376 patients with T2DM were seen in the family medicine outpatient clinic. About $41.5 \%$ of patients with pre-diabetes and $34.7 \%$ of those with T2DM had registered for the web portal (Table 1). Black patients with pre-diabetes or T2DM were less likely to have registered for the web portal than their white counterparts. Diabetic patients who were 18 to 25 years of age were less likely to have registered for the web portal, along with those who were $>65$ years of age. Pre-diabetic patients who were $>65$ years of age were less likely to have registered for the web portal than younger patients with pre-diabetes.

Tables 2 and 3 show the relationship between race and sex in patients with T2DM and pre-diabetes, respectively. Among patients with T2DM but not pre-diabetes, race appeared to modify the relationship between sex and patient web portal registration, but the effect modification on an additive scale was not statistically significant (Tables 2,3). White women with T2DM were more likely to have registered for the web portal than Black women or Black men with T2DM (Table 2). White women with pre-diabetes were more likely to have registered for the web portal than Black women or Black men with pre-diabetes (Table 3).

Table 4 summarizes results from logistic regression analysis of variables associated with web portal registration among patients with T2DM. Statistically significant associations were observed between patient web portal registration and age 41-45 years, and with Hispanic ethnicity. Race was found to modify the association between sex and patient web portal association on a multiplicative scale (Table 4). Web portal registration was less frequent among Black men and Black women with T2DM as compared with their white counterparts. Similar results were obtained for pre-diabetes except that the positive association with age 41-45 years and inverse association with Hispanic ethnicity were not statistically significant (Table 5). 
Table 1 Numbers of diabetic and pre-diabetic patients who registered for the web portal by age, sex, race, and Hispanic ethnicity

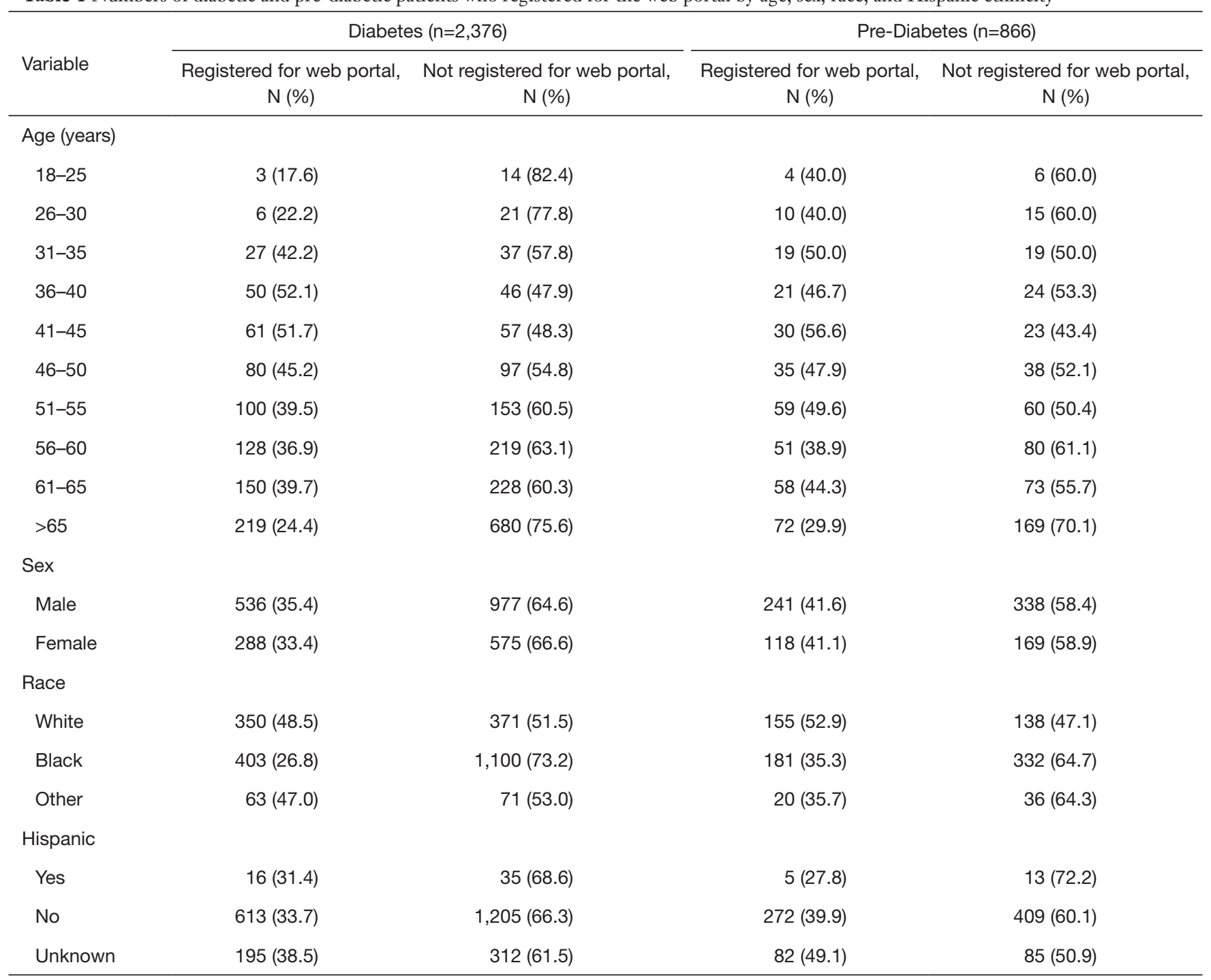

Table 2 Numbers of adult patients with type 2 diabetes mellitus who registered for the web portal by race and sex*

\begin{tabular}{lccc}
\hline Demographic variables & Registered for web portal, N (\%) & Not registered for web portal, N (\%) & OR (P value) \\
\hline White female & $201(50.3)$ & $199(49.8)$ & $3.42(0.000)$ \\
Black female & $293(28.7)$ & $728(71.3)$ & $1.36(0.017)$ \\
White male & $149(46.4)$ & $172(53.6)$ & $2.93(0.000)$ \\
Black male & $110(22.8)$ & $372(77.2)$ & $1.00^{\dagger}(-)$ \\
\hline
\end{tabular}

*, Because the numbers of patients were small, those whose race was 'Other' were excluded from this analysis along with those who were Hispanic. Chi-square test for additive interaction not significant $(\mathrm{P}>0.05) ;{ }^{\dagger}$, referent category. OR, odds ratio. 
Table 3 Numbers of adult patients with pre-diabetes who registered for the web portal by race and sex*

\begin{tabular}{lccc}
\hline Demographic variables & Registered for web portal, N (\%) & Not registered for web portal, N (\%) & OR (P value) \\
\hline White female & $84(51.5)$ & $79(48.5)$ & $2.67(0.000)$ \\
Black female & $142(37.8)$ & $234(62.2)$ & $1.52(0.052)$ \\
White male & $71(54.6)$ & $59(45.4)$ & $3.02(0.000)$ \\
Black male & $39(28.5)$ & $98(71.5)$ & $1.00^{\dagger}(-)$ \\
\hline
\end{tabular}

*, Because the numbers of patients were small, those whose race was 'Other' were excluded from this analysis along with those who were Hispanic. Chi-square test for additive interaction not significant $(\mathrm{P}>0.05) ;{ }^{\dagger}$, referent category. OR, odds ratio.

Table 4 Logistic regression analysis of variables associated with web portal registration among adult clinic patients with type 2 diabetes mellitus ( $\mathrm{n}=2,358)$

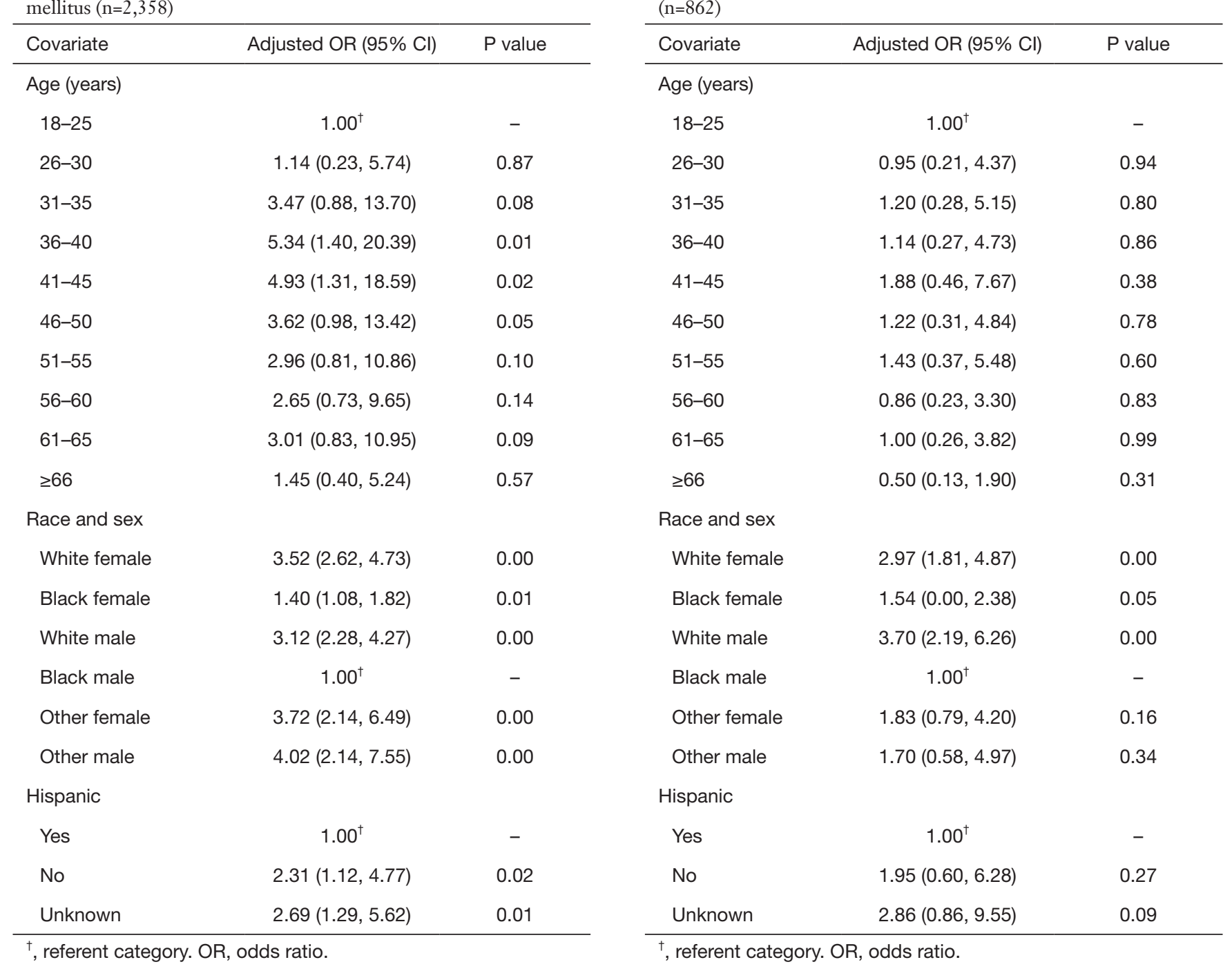

Table 5 Logistic regression analysis of variables associated with web portal registration among adult clinic patients with pre-diabetes (n=862) 


\section{Conclusions}

To our knowledge, this is the first study of web portal use by patients with pre-diabetes. The results of this feasibility study indicate that relatively few adult clinic patients with pre-diabetes have registered for the web portal and that registration rates are particularly low among patients who are Black or aged $>65$ years. The results of this study also indicate that relatively few adult clinic patients with T2DM have registered for the web portal and that registration rates are particularly low among patients who are Black, or aged $<26$ or $>65$ years. Based upon these findings, we are planning larger and more detailed studies to determine why many patients do not register for or use the web portal and to examine the effectiveness of the web portal in improving health outcomes among adult clinic patients with T2DM or pre-diabetes. A goal of the planned research, which is dependent upon receipt of extramural funding, is to determine the specific portal features that may improve quality of care or glycemic control (e.g., patient-provider secure messaging) and to examine the effectiveness of an intervention to increase use of the patient web portal by T2DM patients.

In a previous study of web portal use among adult clinic patients with T2DM see in General Internal Medicine and Endocrinology outpatient clinics, in which patients are provided with access to the Augusta Health Virtually Informed Patient (VIP) web portal, we also observed disparities in web portal use by Black race and age (16). The inverse association with Black race may be due to uncontrolled confounding by health literacy (17-19). In the current feasibility study, no information was available about health literacy of the adult patients with prediabetes or T2DM. Limited the health literacy is relatively common among patients with type 2 diabetes and is likely to contribute to poorer diabetes outcomes (17-21). Studies indicate that patients with lower health literacy are less likely to use web portals to help manage a chronic illness $(13,17,19)$. Patients with lower health literacy or computer literacy may have difficulty learning how to use a web portal or they may have difficulty navigating a portal. Educational outreach efforts for potential web portal users may be helpful in overcoming these barriers.

A limited number of studies have examined the use of web portals tethered to EMRs for diabetes management (22). Although results-to-date have been mixed, results from prior studies suggest that secure messaging between adult diabetic patients and their clinician is associated with improved glycemic control (22). Improvements in low density lipoprotein (LDL) cholesterol and blood pressure have also been observed in some studies of web portals used by patients with diabetes and hypertension (7). The specific portal features that may impact quality of care or improve glycemic control (e.g., patient-provider secure messaging, online access to lab test results or instructions, other patient supports) have not been clearly distinguished by studies completed to date. Results from prior studies indicate that many diabetic patients do not take advantage of web portal features such as secure messaging, when offered, perhaps because of a lack of internet access or to a lack of experience in navigating web portal resources (18).

With respect to limitations, the current study did not examine the specific reasons that patients with pre-diabetes or T2DM had registered for the web portal, whether they used it over time, and the frequency of use. Future studies should examine whether patients who have registered for the web portal use it for secure messaging and whether they are active secure message users or whether they only rarely send secure messages to their provider. A further limitation is that the current study was cross-sectional in nature and lacked information about the temporal relationship between patient web portal registration and improved selfmanagement and better glycemic control.

Additional research is needed to identify specific portal design features that improve glycemic control and interventions that are effective in increasing use of this patient web portals among diabetic and pre-diabetic patients, especially among Black patients, older patients, and among those with low health literacy.

\section{Acknowledgments}

The authors are grateful to Patrick Hatch for providing assistance with the abstraction of information from electronic medical records.

Funding: None.

\section{Footnote}

Conflicts of Interest: All authors have completed the ICMJE uniform disclosure form (available at http://dx.doi. org/10.21037/jhmhp.2018.04.04). The contents do not represent the views of the U.S. Department of Veterans Affairs or the U.S. Government. The authors have no conflicts of interest to declare. 
Ethical Statement: The authors are accountable for all aspects of the work in ensuring that questions related to the accuracy or integrity of any part of the work are appropriately investigated and resolved. The study was conducted in accordance with the Declaration of Helsinki (as revised in 2013). The current study was conducted as "preparatory to research" in support of grant applications for larger and more detailed proposed studies. Prior to a review of computerized patient records, a determination was made that the study was exempt from IRB review. Informed consent was not required for a review of existing patient records that did not include any personally identifying information.

Open Access Statement: This is an Open Access article distributed in accordance with the Creative Commons Attribution-NonCommercial-NoDerivs 4.0 International License (CC BY-NC-ND 4.0), which permits the noncommercial replication and distribution of the article with the strict proviso that no changes or edits are made and the original work is properly cited (including links to both the formal publication through the relevant DOI and the license). See: https://creativecommons.org/licenses/by-nc-nd/4.0/.

\section{References}

1. CDC. National Diabetes Statistics Report, 2017. Estimates of Diabetes and Its Burden in the United States. Available online: https://www.cdc.gov/diabetes/pdfs/data/statistics/ national-diabetes-statistics-report.pdf

2. Bullard KM, Saydah SH, Imperatore G, et al. Secular changes in U.S. Prediabetes prevalence defined by hemoglobin A1c and fasting plasma glucose: National Health and Nutrition Examination Surveys, 1999-2010. Diabetes Care 2013;36:2286-93.

3. CDC. Diabetes. Working to Reverse the US Epidemic, At A Glance 2016. Available online: https://www.cdc.gov/ chronicdisease/resources/publications/aag/diabetes.htm

4. Ali MK, Bullard KM, Saaddine JB, et al. Achievement of goals in U.S. diabetes care, 1999-2010. N Engl J Med 2013;368:1613-24. Erratum in: N Engl J Med 2013;369:587.

5. Saydah SH, Fradkin J, Cowie CC. Poor control of risk factors for vascular disease among adults with previously diagnosed diabetes. JAMA 2004;291:335-42.

6. Gaede P, Vedel P, Larsen N, et al. Multifactorial intervention and cardiovascular disease in patients with type 2 diabetes. N Engl J Med 2003;348:383-93.

7. Shimada SL, Allison JJ, Rosen AK, et al. Sustained Use of Patient Portal Features and Improvements in Diabetes Physiological Measures. J Med Internet Res 2016;18:e179.

8. Heisler M, Piette JD, Spencer M, et al. The relationship between knowledge of recent HbA1c values and diabetes care understanding and self-management. Diabetes Care 2005;28:816-22.

9. Berikai P, Meyer PM, Kazlauskaite R, et al. Gain in patients' knowledge of diabetes management targets is associated with better glycemic control. Diabetes Care 2007;30:1587-9.

10. Anderson RM, Funnell MM, Butler PM, et al. Patient empowerment. Results of a randomized controlled trial. Diabetes Care 1995;18:943-9.

11. Rachmani R, Levi Z, Slavachevski I, et al. Teaching patients to monitor their risk factors retards the progression of vascular complications in high-risk patients with Type 2 diabetes mellitus--a randomized prospective study. Diabet Med 2002;19:385-92.

12. Ricciardi L, Mostashari F, Murphy J, et al. A national action plan to support consumer engagement via e-health. Health Aff (Millwood) 2013;32:376-84.

13. Coughlin SS, Prochaska JJ, Williams LB, et al. Patient web portals, disease management, and primary prevention. Risk Manag Healthc Policy 2017;10:33-40.

14. Schlesselman JJ. Case-control studies: design, conduct, analysis. New York: Oxford University Press, 1982.

15. Snedecor GW, Cochran WG. Statistical methods, 6th ed. Ames, LA: Iowa State University Press, 1967.

16. Coughlin SS, Heboyan V, Williams LB, et al. Use of a web portal among adult clinic patients seen for type 2 diabetes mellitus. J Hosp Manag Health Policy 2018;2. pii: 1.

17. Sarkar U, Karter AJ, Liu JY, et al. The literacy divide: health literacy and the use of an internet-based patient portal in an integrated health system-results from the diabetes study of northern California (DISTANCE). J Health Commun 2010;15 Suppl 2:183-96.

18. Wade-Vuturo AE, Mayberry LS, Osborn CY. Secure messaging and diabetes management: experiences and perspectives of patient portal users. J Am Med Inform Assoc 2013;20:519-25.

19. Coughlin SS, Stewart JL, Young L, et al. Health literacy and patient web portals. Int J Med Inform 2018;113:43-8.

20. Morris NS, MacLean CD, Littenberg B. Literacy and health outcomes: a cross-sectional study in 1002 adults with diabetes. BMC Fam Pract 2006;7:49. 
21. Cavanaugh $\mathrm{K}$, Huizinga MM, Wallston KA, et al. Association of numeracy and diabetes control. Ann Intern Med 2008;148:737-46.

doi: 10.21037/jhmhp.2018.04.04

Cite this article as: Coughlin SS, Heboyan V, Young L, De Leo G, Wilkins T. Use of a web portal by adult patients with prediabetes and type 2 diabetes mellitus seen in a family medicine outpatient clinic. J Hosp Manag Health Policy 2018;2:21.
22. Coughlin SS, Williams LB, Hatzigeorgiou C. A systematic review of studies of web portals for patients with diabetes mellitus. Mhealth 2017;3:23. 